\title{
AN OVERVIEW OF STACK DESIGN FOR A THERMOACOUSTIC REFRIGERATOR
}

\author{
Bhansali P. S ${ }^{1}$, Patunkar P. $P^{2}$, Gorade S. $V^{3}$, Adhav S. $S^{4}$, Botre S. $S^{5}$ \\ ${ }^{1,2,3,4}$ Student, Department of Mechanical Engineering, Sinhgad Institute of Technology \& Science, Pune, Maharashtra, \\ India \\ ${ }^{5}$ Assistant Professor, Department of Mechanical Engineering, Sinhgad Institute of Technology \& Science, Pune, \\ Maharashtra, India
}

\begin{abstract}
A thermoacoustic refrigerator utilizes the thermal interactions of the sound waves with the medium while they travel to produce refrigeration. Sound energy propagates in longitudinal fashion through the medium, thus resulting in compressions and rarefactions in the medium and hence heating and cooling the medium subsequently. The stack acts as a medium to transfer the heat from one point in the system to another. The stack is thus the heart of any thermoacoustic refrigeration system. This paper provides a brief overview of the construction and working of the thermoacoustic refrigerator and focusses on the stack of a thermoacoustic refrigeration system. The desired thermal properties of the stack material like the thermal conductivity and specific heat have been discussed. An optimum spacing obtained from previous works based on the thermal and viscous penetration depths has been briefly discussed. Various stack geometries like the parallel plate type, the spiral type, pin type and porous stacks made of reticulated vitreous carbon have been elaborated.
\end{abstract}

Keywords: Thermoacoustic refrigerator, Stack, Thermal penetration depth, Stack geometry, Stack spacing. $* * *$

\section{INTRODUCTION}

Thermoacoustic refrigeration is an eco-friendly refrigeration method which employs sound waves to produce cooling. It overcomes the drawbacks of conventional methods like the vapour compression system, air refrigeration system, etc., which require harmful refrigerants and moving parts like compressor and hence, is more reliable. The field of thermoacoustics has attracted researchers from 1816 when Pierre-Simon Laplace took into account the local change in temperature and pressure due to the compression and rarefaction that takes place in the medium during sound propagation [1]. $19^{\text {th }}$ century glass blowers used to hear pure tones emitted by their heated vessels. The reverse phenomenon was recognised after more than a century and it was realised that sound could conversely produce a temperature difference [2]. Notable advances were done after the work of P. Rijke, who employed a glass tube open at both closings and a wire mesh to locally heat the air. Tone close to the fundamental tone of the glass tube was obtained [3]. In a similar experiment, Lord Rayleigh used two gauze layers, the extra one to retain heat for a longer time. This reportedly produced strong oscillations which shook the room [4]. N. Rott made a considerable contribution by modelling of thermodynamic phenomenon through his linear theory [5]. He laid the mathematical foundation for thermoacoustics [6]. The field was further galvanized by notable contributions from G. Swift, who associated it with the broader field of thermodynamics [7]. Sound waves in air are longitudinal waves. The medium in which they move undergoes vibrations, thus experiences compression and rarefaction. This is associated with change in temperature and pressure. When the gas carrying a wave is brought in contact with a solid surface, it absorbs the heat as the gas gets compressed. Since the specific heat capacity of solids is generally quite greater than that of fluids, the solid absorbs heat without much change in its temperature. Similarly, it rejects heat to the gas molecules nearby during expansion, thus maintaining stable temperature. It is obvious that large amplitudes of pressure lead to an increase in heat transfer since it generates larger temperature difference between the gas and the solid.

If continuous constant frequency vibration (by means of sound waves) is supplied to the gas in a tube with one end (reflector end) close, the reflected and the originally supplied sound waves will interfere with each other. If the length of the tube is selected so as to obtain resonance, a standing wave is obtained. The pressure amplitude thus obtained is high and can be used to produce temperature difference across the tube. In the case of tube with one end open, length of tube required to sustain resonance should be equal to odd integrals of $\lambda / 4$.

\section{CONSTRUCTION}

Fig-1 shows the simple schematic of a standing wave thermoacoustic refrigerator [8]. It consists of a resonance tube which contains a suitable working gas. Aluminium plug is inserted at one end to close it. This plug also acts as a reflector surface. A loudspeaker is used to generate acoustic vibrations in the medium. A porous medium called the stack is placed in the tube to increase the gas solid interface discussed earlier and hence enhance heat exchange. The temperature difference is to be generated across this stack. A thermocouple is used at both ends of the stack so as to measure the temperatures and hence also the temperature difference generated across the stack. The area of the 
loudspeaker diaphragm which is not covered by the resonating tube is closed with the help of a covering plate. This avoids the loss of acoustic power to the surrounding through the opening. O rings are used to seal the gap between the loudspeaker box and the resonator tube so as to avoid any leakage.

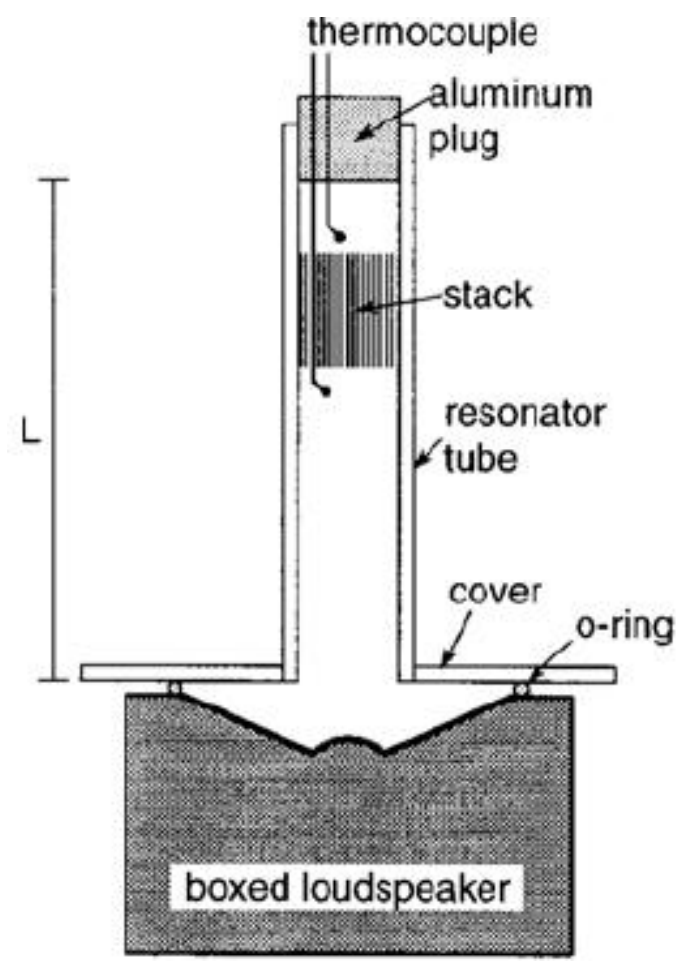

Fig-1: Schematic representation of TAR [8].

\section{WORKING}

When a constant frequency tone is generated by the loudspeaker, a standing wave is obtained in the tube. Consider a gas packet oscillating in the medium. As the gas packet expands due to propagation of sound, its temperature falls. It absorbs heat from the stack surface in the vicinity, thus producing a drop in temperature. As the gas parcel now moves to the high pressure regions, its temperature rises and it rejects heat to the stack material at this point. Thus the pumping of heat takes place.

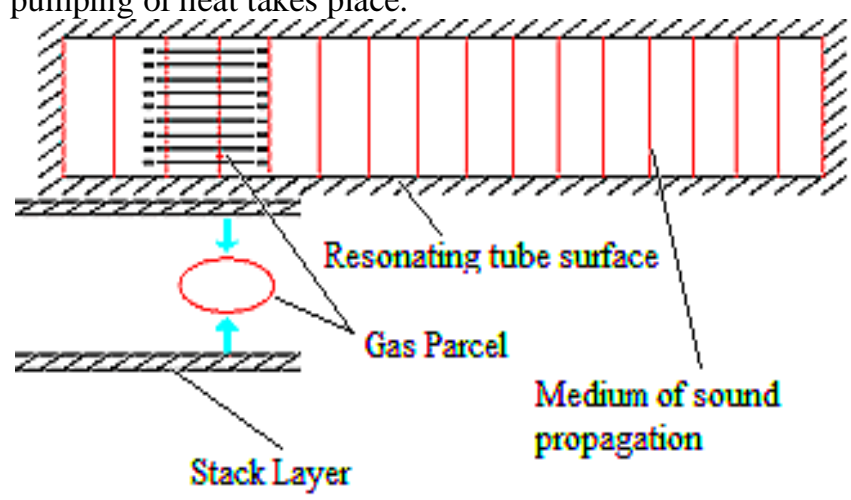

(a)

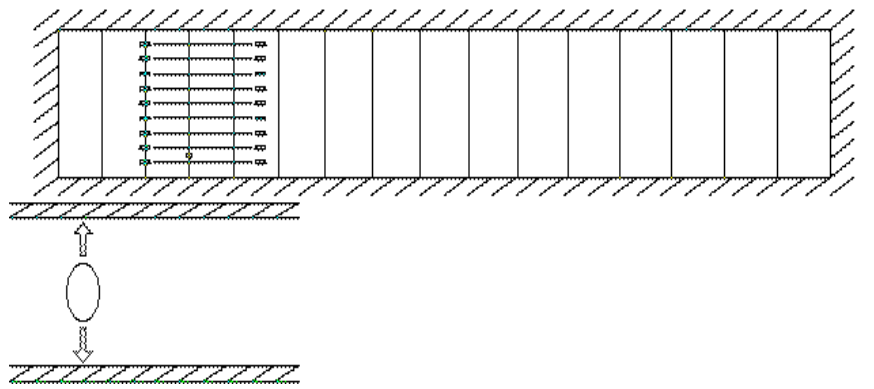

(b)

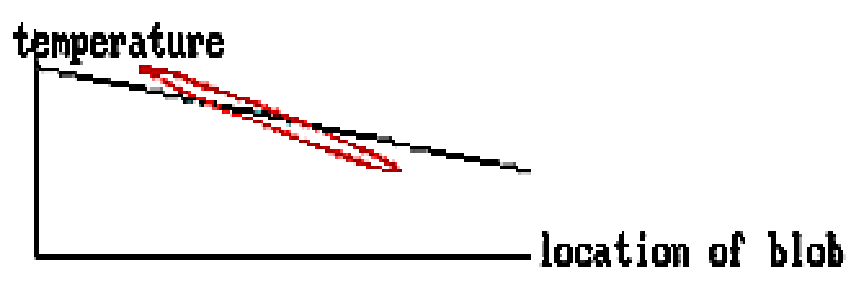

(c)

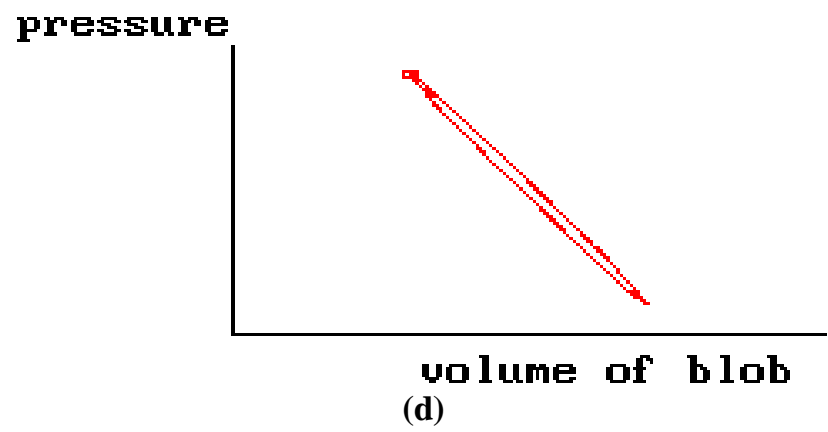

Fig-2: Gas parcel during various stages of the cycle and the direction of heat transfer. (a): The gas parcel during expansion. Heat is absorbed from the stack by the cold gas parcel. (b): The gas parcel during compression. Heat is rejected to the stack by the heated gas parcel. (c): Variation of temperature of gas parcel with its location in the stack.

(d): Variation of pressure of the gas parcel with its volume.

Fig-2 shows a gas parcel undergoing various stages in the cycle as prescribed by G. Swift [9] and obtained from the animations provided by Los Alamos National Laboratory [10]. The gas oscillates between two displacement nodes only. During the oscillation, when it undergoes expansion, its temperature drops. It thus absorbs heat from the stack locally in its vicinity. As the blob begins to compress while moving towards the other end of the stack, its temperature rises and it rejects heat to the stack surface in contact. Thus heat is pumped from one end of the stack to the other.

\section{LITERATURE REVIEW}

A stack is the heart of a thermoacoustic refrigeration system. The stack provides increased gas solid interface and makes it possible to generate adequate temperature difference. The absence of stack would imply heat pumping only from the tube surface rendering the system ineffective. This paper aims at highlighting various analysis done for parameters of the stack. 


\subsection{Stack Material}

The thermal conductivity of the stack material has a negative impact on the performance of the thermoacoustic refrigerator [11] [12]. If the thermal conductivity of the stack material is high, the heat which has been pumped by the system will be conducted back to the cold side, thus reversing the cooling effect. Thus, a stack essentially must be made up of a material with low thermal conductivity. The material Mylar which has a thermal conductivity $0.16 \mathrm{~W} / \mathrm{m}$ $\mathrm{K}$ [13] is widely used. Adef et al. [14]

The heat capacity of stack material should be quite higher than that of the gas so that the temperature remains steady [7].

\subsection{Thermal and Viscous Penetration Depths}

Thermal penetration depth is the distance in the gas from stack surface up to which the heat the heat can diffuse. It indicates the region in gas where the molecules take part in the thermoacoustic effect [15]. The thermal penetration depth is given by

$$
\delta_{k}=\sqrt{\frac{2 \kappa}{\rho C_{p} \omega}} \quad \ldots
$$

This thermal penetration depth extends on both sides of the stack material.

However, there are some energy losses near the solid surface. This is because viscous stresses are produced during the oscillations of the fluid [16]. This generally occurs in the volume up to distance from the surface equal to the viscous penetration depth [15] given by

$$
\delta_{v}=\sqrt{\frac{2 \mu}{\rho \omega}} \quad \ldots
$$

Where, $\kappa=$ thermal conductivity of the gas

$$
\begin{array}{ll}
\rho & =\text { density of the gas } \\
C_{p} & =\text { specific heat at constant pressure } \\
\omega & =\text { angular frequency of the sound wave. }
\end{array}
$$

\subsection{Stack Spacing}

Since the thermal penetration depth extends on both the sides of the stack surface, two layers must be so spaced that they do not interfere in each other's thermal penetration depth. So the stack spacing must be at least equal to $2 \delta_{k}$. Its value is suggested to be between $2 \delta_{k}$ to $4 \delta_{k}$ [17].

Tijani et al. [13] investigated the effect of stack spacing on the performance of a thermoacoustic refrigerator. Various stacks with plate spacing ranging from 0.15 and $0.7 \mathrm{~mm}$ were manufactured and analysed. The variation of performance parameters with the stack spacing $2 y_{0}$, was studied.

Where, $\mathrm{y}_{\mathrm{o}} \quad=$ half stack spacing.

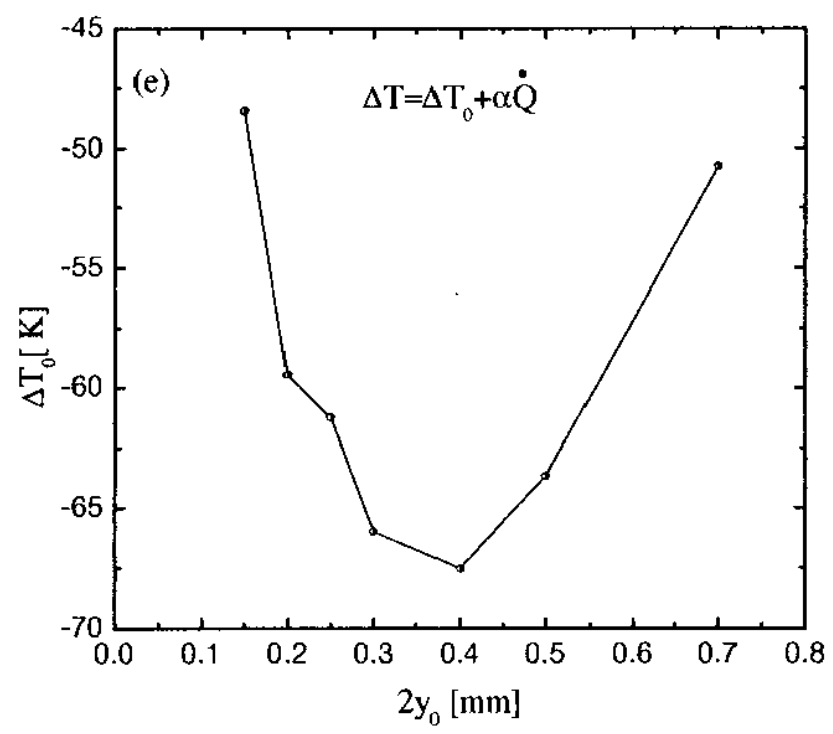

(a)

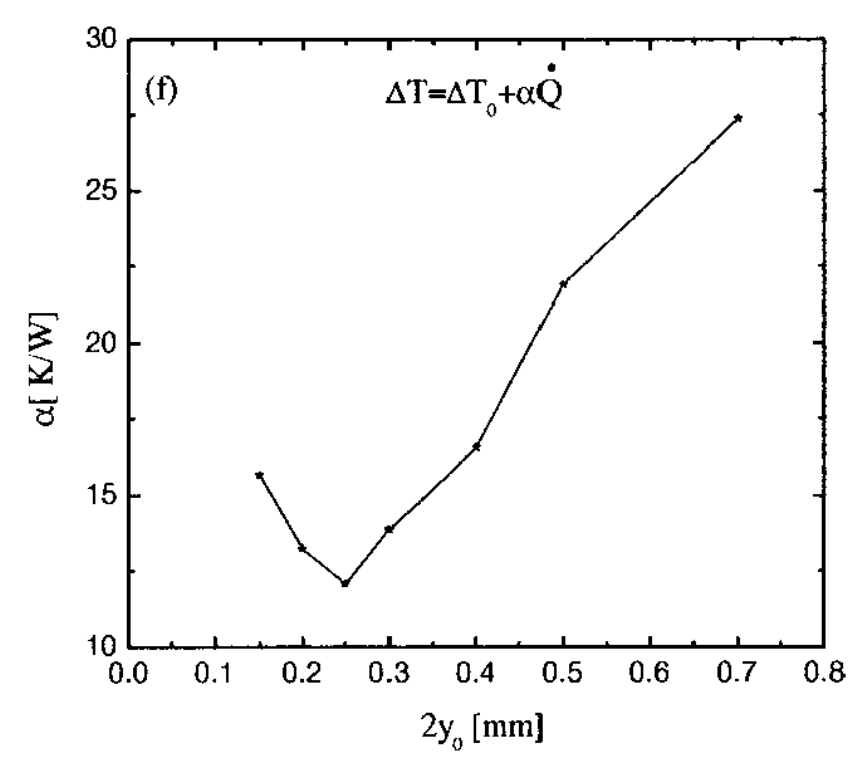

(b)

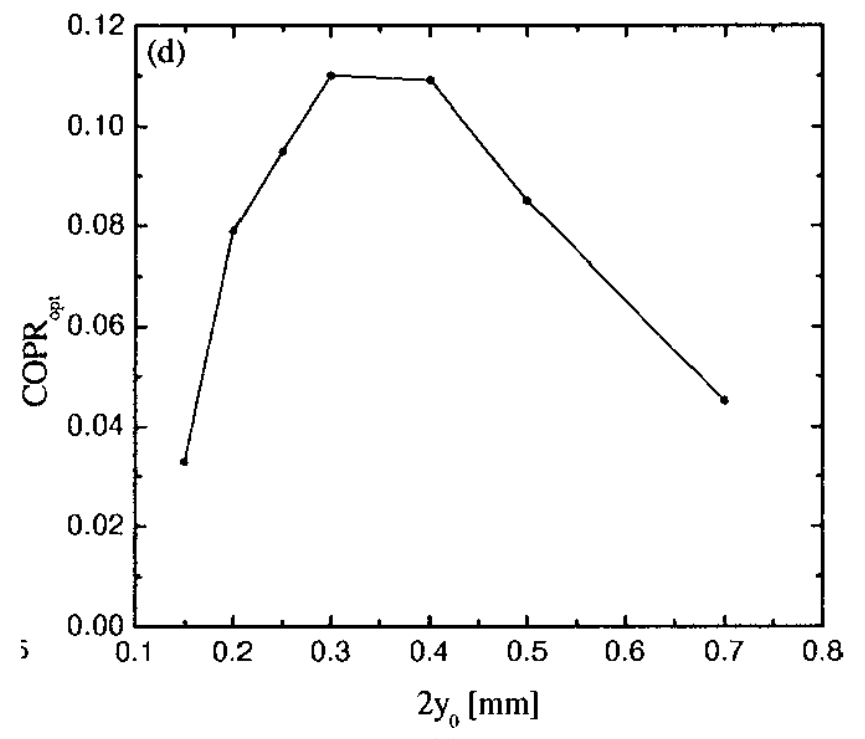

(c) 
Fig-3: Effect of stack spacing on the performance of thermoacoustic refrigerator. (a): Temperature difference without heat load $\left(\Delta \mathrm{T}_{\mathrm{o}}\right)$ vs. $2 \mathrm{y}_{\mathrm{o}}$. (b): Coefficient a vs. $2 \mathrm{y}_{\mathrm{o}}$. (c): COPR vs. $2 \mathrm{y}_{\text {o. }}[13]$

It can be observed in fig-3(a) that the temperature difference without heat load is optimum for a stack spacing of $0.4 \mathrm{~mm}$ $\left(4 \delta_{k}\right)$ and fig-3(b) indicates maximum cooling power at a spacing of $2.5 \delta_{k}$. It can be seen from fig-3(c) that the maximum COPR is obtained at $3 \delta_{k}$. Tijani et al. suggested a stack spacing of $3 \delta_{k}$.

\subsection{Stack Geometry}

Stacks of various geometries like the parallel plate type, spiral type, pin-array type have been developed and studied over the years so as to get improves performance, simplicity of manufacturing and cost effectiveness. Some geometries are discussed in this paper.

A parallel plate stack consists of plates of the stack material placed parallel to each other and separated by separator linings.

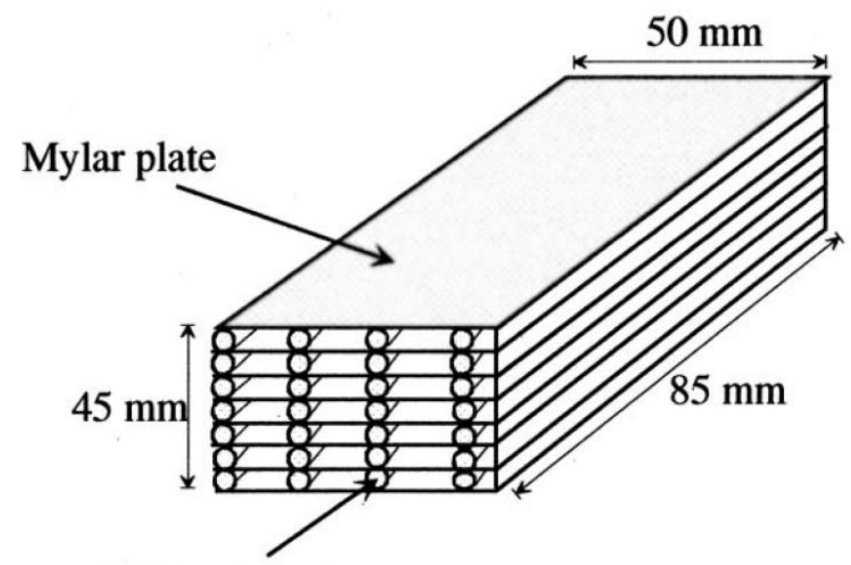

Fishing line

Fig-4: Parallel plate stack [13].

Tijani et al. [13] used Mylar material to construct a parallel plate stack as illustrated in fig-4. Nylon fishing lines were used to maintain the stack spacing. A parallel plate stack is, however, difficult to manufacture.

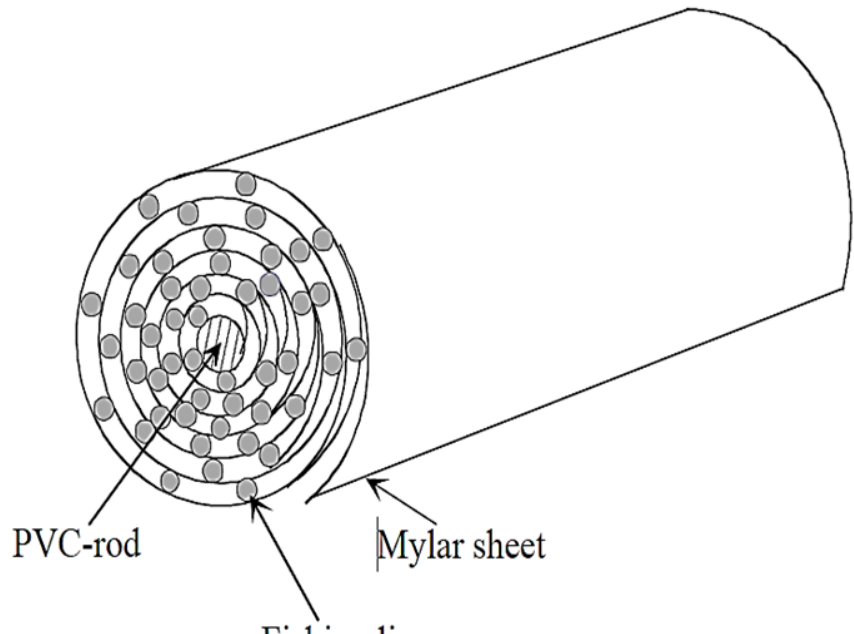

Fishing line

Fig-5: Spiral Stack [11].

Tijani et al. [11] manufactured two spiral stacks make up of Mylar sheet wound around a PVC rod as illustrated in fig-5. Excessive tension in one stack resulted in deformed channels. The other stack was carefully wound.

Adeff et al. [18] performed experiments using a stack made of reticulated vitreous carbon, an open pore foam material made purely of vitreous carbon.

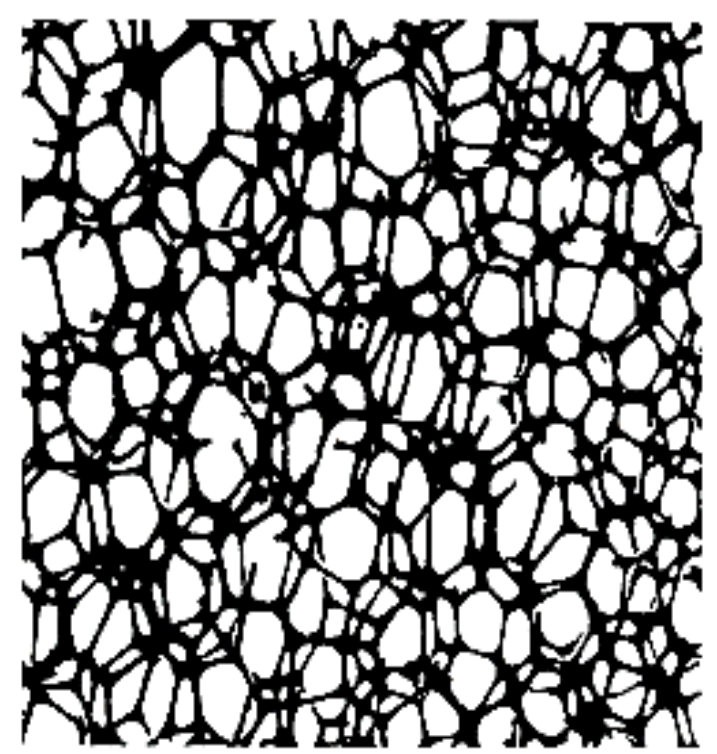

Fig-6: Open Pore Reticulated Structure of RVC [18].

RVC is a glassy rigid sponge like carbon material. The performance of this stack was comparable to that of a plastic roll stack. 
$\mathrm{O}$

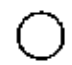

(
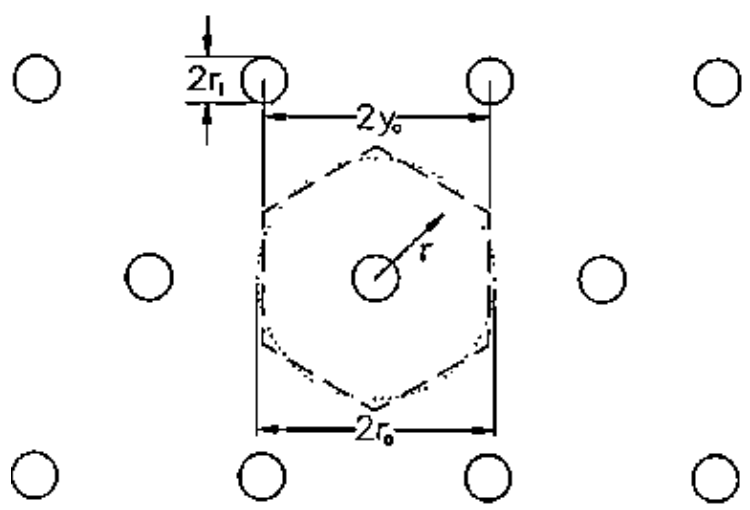

Fig-7: Pin Stack Geometry [16]

Energy is lost due to the viscous interaction of the fluid with the solid surface. Keolin et al. [16] obtained a patent for their pin array structure. This geometry provided a convex cross section perpendicular to the axis of oscillation of the gas. This resulted in an increase in the ratio of volume at the thermal penetration depth to that at the viscous penetration depth, thus increasing the ratio of thermal energy transferred to the viscous losses. Fig-7 shows the pin stack geometry using pins of radius $r_{i}$. The spacing between nearest neighbour pins is $2 y_{0}$. The hexagonal unit cell assumed is shown by dotted lines.

\section{CONCLUSION}

The performance of a thermoacoustic refrigerator greatly depends on the stack used. The stack material must have low thermal conductivity and higher heat capacity than the acoustic medium. Also, the stack geometry affects the performance of the refrigerator. Stack spacing is an important consideration in the stack geometry. Too low spacing leads to higher viscous losses and too larger spacing results in only a small volume of gas involved in the thermal interaction. A stack spacing of 3 times the thermal penetration depth is suggested. Stacks of various shapes have been designed. The spiral stack is the simplest to design. The pin stack reduces viscous losses and offers an improved ratio of energy transmitted to the viscous losses.

\section{REFERENCES}

[1] Finn, Bernard S. "Laplace and the Speed of Sound." Isis (1964): 7-19.

[2] Garrett, Steven L., and Scott Backhaus. "The Power of Sound." American Scientist 88 (2000).

[3] Rijke, P. L. "LXXI. Notice of a new method of causing a vibration of the air contained in a tube open at both ends." The London, Edinburgh, and Dublin Philosophical Magazine and Journal of Science 17.116 (1859): 419-422.

[4] Rayleigh, Lord. "XXVII. Acoustical observations. II." The London, Edinburgh, and Dublin Philosophical Magazine and Journal of Science 7.42 (1879): 149-162.
[5] Rott, Nikolaus. "Damped and thermally driven acoustic oscillations in wide and narrow tubes." Zeitschrift für angewandte Mathematik und Physik ZAMP 20.2 (1969): 230-243.

[6] Rott, Nikolaus. "Thermoacoustics." Advances in applied mechanics 20 (1980): 135-175.

[7] Swift, G. W., and Steven L. Garrett. "Thermoacoustics: a unifying perspective for some engines and refrigerators." The Journal of the Acoustical Society of America 113 (2003): 23792381.

[8] Doshi, Dev, et al. "Thermoacoustic Refrigeration."

[9] Swift, G. W. "Thermoacoustics: A unifying perspective for some engines and refrigerators. 2002." Melville, NY: Acoustical Society of America: 300.

[10] N.p., n.d. Web. http://www.lanl.gov/thermoacoustics/movies.html

[11] Tijani, Moulay El Hassan. Loudspeaker-driven thermo-acoustic refrigeration. Eindhoven, Netherlands: Technische Universiteit Eindhoven, 2001.

[12] Swift, Gregory W. "Thermoacoustic engines." The Journal of the Acoustical Society of America 84.4 (1988): 1145-1180.

[13] Tijani, M. E. H., J. C. H. Zeegers, and A. T. A. M. De Waele. "The optimal stack spacing for thermoacoustic refrigeration." The Journal of the Acoustical Society of America 112.1 (2002): 128133.

[14] Adeff, Jay A., et al. "Measurements with reticulated vitreous carbon stacks in thermoacoustic prime movers and refrigerators." The Journal of the Acoustical Society of America 104.1 (1998): 32-38.

[15] Tijani, M. E. H., J. C. H. Zeegers, and A. T. A. M. De Waele. "The optimal stack spacing for thermoacoustic refrigeration." The Journal of the Acoustical Society of America 112.1 (2002): 128133.

[16] Keolian, Robert M., and Gregory W. Swift. "Pin stack array for thermoacoustic energy conversion." U.S. Patent No. 5,456,082. 10 Oct. 1995.

[17] Tijani, Moulay El Hassan. Loudspeaker-driven thermo-acoustic refrigeration. Eindhoven, Netherlands: Technische Universiteit Eindhoven, 2001.

[18] Adeff, Jay A., et al. "Measurements with reticulated vitreous carbon stacks in thermoacoustic prime movers and refrigerators." The Journal of the Acoustical Society of America 104.1 (1998): 32-38. 